\title{
FKBP5 as a possible moderator of the psychosis-inducing effects of childhood trauma
}

Dina Collip, Inez Myin-Germeys, ${ }^{*}$ Marieke Wichers, ${ }^{*}$ Nele Jacobs, Catherine Derom, Evert Thiery, Tineke Lataster, Claudia Simons, Philippe Delespaul, Machteld Marcelis, Jim van Os and Ruud van Winkel

\section{Background}

FK506 binding protein 5 (FKBP5) has repeatedly been shown to be a critical determinant of post-traumatic stress disorder (PTSD) and depression following childhood trauma.

\section{Aims}

To examine the role of FKBP5-trauma interactions in the partly stress-related psychosis phenotype.

\section{Method}

In 401 general population twins, four functional polymorphisms were examined in models of psychosis and cortisol, and followed up in models of psychosis in three samples at different familial liability (175 controls, 200 unaffected siblings and 195 patients with a psychotic disorder).

\section{Results}

The most consistent finding was an interaction between childhood trauma and rs9296158/rs4713916 on psychotic symptoms and cortisol in the twin sample, combined with a directionally similar interaction in siblings ( $r$ s4713916) and patients (rs9296158), A-allele carriers at both polymorphisms being most vulnerable to trauma.

\section{Conclusions}

Trauma may increase the risk of psychosis through enduring changes in the cortisol feedback loop, similar to that for PTSD, suggesting comparable biological mechanisms for psychosis across diagnostic boundaries.

\section{Declaration of interest}

None.
Meta-analytic evidence suggests that childhood trauma plays an important role in the development of psychotic outcomes, both at the level of psychotic disorder and at the level of the extended psychosis phenotype of subclinical psychotic experiences in the general population. ${ }^{1}$ A recent study investigated patients with psychotic disorder, their healthy siblings and controls, and found that the association between trauma and psychosis was apparent across different levels of illness and vulnerability to psychotic disorder, suggesting a true association rather than reporting bias, reverse causality or a passive gene-environment correlation. ${ }^{2}$ However, although childhood trauma may have true aetiological relevance, it is neither necessary nor sufficient for the development of psychosis and other factors such as underlying genetic liability are likely to also play a role. ${ }^{3}$

The causal role of childhood trauma may be mediated in part by alterations in hypothalamic-pituitary-adrenocortical (HPA) axis functioning. ${ }^{4}$ Several lines of evidence indicate that dysregulation of the HPA axis may play a role in the relationship between a stressful environment (for example trauma) and psychotic experiences ${ }^{3}$ in individuals with a psychotic disorder or at increased risk of developing a psychotic disorder. ${ }^{5}$ Abnormalities have been found at various levels of the HPA axis, including functional and structural changes in the pituitary and hippocampus. ${ }^{6}$ Similarly, dysregulation of cortisol and, possibly, abnormalities in glucocorticoid receptors may be present. Furthermore, there may be a synergistic relationship between activation of the HPA axis and activation of dopaminergic circuits that have been implicated in psychosis. Although the exact mechanisms remain to be elucidated, evidence suggests that glucocorticoid secretion may increase dopamine activity in brain regions that have been associated with experience of positive psychotic symptoms. ${ }^{7}$

\footnotetext{
*These authors contributed equally to the work.
}

The data thus suggest that glucocorticoid signalling may play a pivotal role in the link between trauma-induced HPA-axis dysregulation and psychosis. Consequently, genetic variation determining functioning of glucocorticoid signalling may explain differential sensitivity to trauma. However, identification of molecular-genetic variants within the context of a geneenvironment $(\mathrm{G} \times \mathrm{E})$ interaction has proven difficult, and recent studies have challenged 'established' interactions such as COMT and cannabis in the field of psychosis, ${ }^{8}$ and serotonin transporter $\times($ social $)$ stress in affective disorders. ${ }^{9}$ An exception to this rule may be the interaction between single nucleotide polymorphisms (SNPs) in the FK506 binding protein 5 (FKBP5) and childhood trauma in their effect on stress-related mental disorders such as affective disorders and post-traumatic stress disorder (PTSD), for which relatively consistent findings were reported, although it should be noted that no formal metaanalysis critically evaluating the entire evidence base is currently available. FK506 binding protein 5 is a critical modulator of the feedback loop determining glucocorticoid receptor sensitivity. Both in children and in adults, FKBP5 SNPs have been associated with symptoms of PTSD when individuals have been exposed to trauma. ${ }^{10,11}$ Given the status of psychotic disorder as a partly stress-related condition, and the relatively consistent FKBP $5 \times$ trauma interactions reported in mood and anxiety phenotypes, the present study aimed to investigate the FKBP $5 \times$ childhood trauma interaction in relation to psychosis. It has been proposed that $\mathrm{G} \times \mathrm{E}$ interactions may be captured best by studying both clinical and subclinical expression of psychosis outcomes. Subthreshold phenomena may better capture the genetic vulnerability that is shared between the clinical and the non-clinical phenotypes ${ }^{12}$ and may be less biased by secondary factors related to the disorder or its treatment; associations at the level of the disorder phenotype confirm the hypothesised clinical relevance. In addition, consistency of interactions across different samples and statistical approaches may be a fruitful strategy to distinguish true interactions from chance findings. ${ }^{13}$ 
Therefore, the current study examined the FKBP $5 \times$ childhood trauma interaction in different samples, across different levels of psychosis severity and genetic liability, and across different phenotypes. The FKBP $5 \times$ trauma interaction was first investigated in a discovery sample of female twins from the general population, at the level of subclinical psychosis (analysis 1) and at the level of biological phenotype (cortisol, analysis 2). Subsequently, in independent replication samples, we investigated whether the FKBP $5 \times$ childhood trauma interaction predicted subclinical expression of psychosis in a healthy control group (analysis 3) and in a sample with elevated genetic risk, i.e. unaffected siblings of patients with psychotic disorder (analysis 4). Finally, we examined the association between FKBP5 SNPs and childhood trauma in the affected probands with psychotic disorder (analysis 5).

\section{Method}

\section{Analyses 1 and 2 - general population twin study}

The discovery sample consisted of 621 female participants, aged 18-61 years, recruited with the purpose to study $\mathrm{G} \times \mathrm{E}$ interactions in mental disorders, as described previously. Participants came from the East Flanders Prospective Twin Survey. This population-based survey has prospectively recorded all multiple births in the province of East Flanders, Belgium, since 1964. ${ }^{14}$ Since the original goal of this sample was to study the role of stress-sensitivity in depression, the sample was female only, given evidence for qualitative differences in the type of environmental stressors that are associated with depression in men and women. Zygosity was determined through sequential analysis based on gender, fetal membranes, blood groups and DNA. Participants were of White ethnic group and of Belgian origin. The Structured Clinical Interview for DSM-IV Axis I disorders (SCID-I) ${ }^{15}$ was administered to obtain current and lifetime diagnoses of major depressive disorder. Subclinical psychosis and depression were assessed using the Community Assessment of Psychic Experiences (CAPE), a validated instrument for assessing subclinical symptoms in general population samples. ${ }^{16}$ Standardised sum scores of the positive and depressive items subscale were used in the analyses. In this sample, as well as in the replication sample analysed in the present paper, mean childhood trauma scores were generated from the Childhood Trauma Questionnaire (CTQ). ${ }^{17}$ The CTQ consists of 25 questions rated on a five-point Likert scale enquiring about traumatic experiences in childhood. In total, 582 individuals completed the trauma measures and of those, 421 participants provided DNA as well. There were no demographic differences between participants included and not included in the analyses regarding age, highest educational level achieved, marital status, work or living situation.

Cortisol was sampled ten times over the course of the day for 5 consecutive days using intrabuccal swaps, a method validated by previous research. ${ }^{18}$ Mean cortisol levels over this period were used for the current analyses. Salivary free cortisol levels were determined in duplicate, using a time-resolved immunoassay with fluorescence detection. ${ }^{19}$ The lower detection limit of this assay was $0.2 \mathrm{nmol} / \mathrm{l}$; interassay and intra-assay coefficients of variation were $<10 \%$. Cortisol samples considered as physiologically abnormal $(>44 \mathrm{nmol} / \mathrm{l})^{18}$ and thus incorrect were therefore excluded from the analysis. The project was approved by the local ethics committee and all participants gave written informed consent.

Multilevel regression analysis was used taking into account clustering within twin pairs using the XTREG command in Stata version 12 for Windows. Significance was assessed by Wald test.
The SNPs were coded 0/1/2 according to minor allele loading and analysed linearly. Age, gender, depressive symptoms and stressful life events were added as a priori confounders. Effect sizes were calculated by combining the appropriate linear combinations using the Stata LINCOM procedure.

Additional confounders for cortisol analyses were oestrogen exposure and, as cortisol alterations are common in depression, participants with a current or lifetime depressive disorder were excluded from the analyses. Power calculations revealed a power of 0.81 (with an anticipated beta of 0.2 for the interaction at an alpha of 0.05 ) for analysis 1 and a power of 0.88 for analysis 2 (with an anticipated beta of 0.25 at an alpha of 0.05). Effect sizes were conservatively estimated based on previous research (estimated based on Appel et $a l^{20}$ Alemany et al ${ }^{21}$ ).

\section{Analysis 3-5 - patients with psychotic disorder, their unaffected siblings and healthy controls}

The sample comprised 272 patients, 258 of their siblings and 227 healthy controls, as described by Heins and colleagues. ${ }^{2}$ Of those, 195 patients, 200 siblings and 175 controls also provided DNA. Analyses 3-5 were therefore conducted in a sample of patients with psychotic disorder $(n=195)$, their unaffected siblings $(n=200)$ and healthy controls $(n=175)$. There were no significant differences in age, IQ, education or ethnicity between the groups of included participants and those who dropped-out; however, more male participants dropped out of the analyses.

In selected representative geographical areas in The Netherlands and Belgium, patients were identified through clinicians working in regional psychotic disorder services, whose case-load was screened for inclusion criteria. Subsequently, patients presenting consecutively at these services either as out-patients or in-patients were recruited for the study. First-degree relatives were sampled through participating patients and control participants were recruited through a system of random mailings in the catchment areas of the cases.

Inclusion criteria were: (a) age 16 to 50 years; (b) diagnosis of non-affective psychotic disorder; and (c) good command of Dutch language. Siblings had to be free of any lifetime non-affective psychotic disorder. For the controls, the occurrence of any psychotic disorder in either the participants or any first-degree family member, assessed using the Family Interview for Genetic Studies (FIGS), ${ }^{22}$ constituted an exclusion criterion. Trained research psychologists based diagnosis on DSM-IV criteria, ${ }^{23}$ assessed with the Comprehensive Assessment of Symptoms and History (CASH) interview. ${ }^{24}$ The DSM-IV diagnoses of the patients were: schizophrenia and related disorders (DSM-IV 295.x: $n=165,84.6 \%$ ), other psychotic disorders (DSM-IV 297/ 298; $n=27,13.8 \%)$ and psychotic illness in the context of substance misuse $(n=3 ; 1.5 \%)$. Subclinical psychotic experiences were defined as the mean of the positive schizotypy subscale of the Structured Interview for Schizotypy - Revised (SIS-R). ${ }^{25}$ The SIS-R is a semi-structured interview containing 20 schizotypal features and 11 schizoptypal signs, rated on a four-point scale. Questions and rating procedures are standardised. Psychotic and depressive symptoms (mean) in the patient sample were assessed with the Brief Psychiatric Rating Scale (BPRS). ${ }^{26}$

Age, gender and depressive symptoms were added as a priori confounders in analyses 3, 4 and 5. In this sample, recent life events were not assessed and could therefore not be added as an a priori confounder, in contrast to analysis 1 in the general population twin sample. Power calculations revealed a power of 0.47 for analysis 3 (with an anticipated beta of 0.2 and alpha of 0.05 , as also applied in analysis 1), a power of 0.72 for 
analysis 4 (with an anticipated beta of 0.25 instead of 0.20 given above-average genetic risk in the unaffected siblings, and alpha of 0.05 ), and a power of 0.87 for analysis 5 (with an estimated beta of 0.3 instead of 0.20 given highest genetic risk in the patients, and alpha of 0.05)..$^{20}$

Moreover, a case-only analysis was considered to examine $\mathrm{G} \times \mathrm{E}$ interactions at the level of the clinical disorder. A case-only design determines presence of $\mathrm{G} \times \mathrm{E}$ interactions on the basis of an association between SNP and exposure, while assuming independence between SNP and exposure. However, a power calculation using Quanto (http://hydra.usc.edu/gxe/) revealed a power of only 0.21 for a case-only analysis when applying an odds ratio (OR) of 5 for the exposed homozygous risk carriers compared with the reference genotype (based on Appel et al ${ }^{20}$ and Alemany et $a l^{21}$ ). Given the unacceptably low power of the case-only design, this analysis was not pursued further.

\section{Genotyping}

In the general population twin sample, four FKBP5 SNPs were chosen based on their documented functional relevance, in terms of mRNA expression or dexamethasone-corticotropin-releasing hormone or dexamethasone suppression effects: rs9296158, rs1043805, rs1360780 and rs4713916. In the replication samples, genotype data for FKBP5 SNPs were available through earlier genotyping efforts. ${ }^{27}$ In this sample, three of the four SNPs were selected for genotyping (rs9296158, rs4713916, rs1360780); however, genotyping failed for rs1360780. Furthermore, five additional SNPs were available in this sample, some of which were in (almost) perfect linkage disequilibrium with the missing functional SNPs (Fig. 1). Thus, rs992105 was used to substitute rs1043805 $\left(r^{2}=1.0\right)$, and rs3800373 was used instead of rs1360780 $\left(r^{2}=0.94\right)$ in the replication efforts. The SNPs were determined by Sequenom (Hamburg, Germany: www.sequenom. $\mathrm{com} /$ ) using the Sequenom MassARRAY iPLEX platform at the facilities of the manufacturer as described previously. ${ }^{27}$ None of
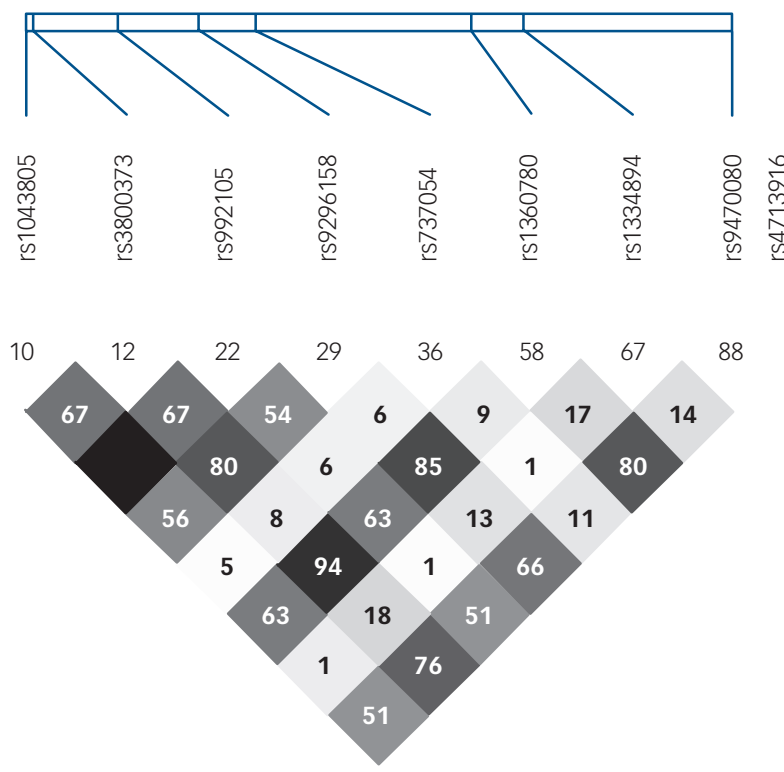

Fig. 1 Position of single nucleotide polymorphisms (SNPS) genotyped within the current studies.

The linkage disequilibrium among the SNPS is depicted as $r^{2}$, with complete linkage disequilibrium, or $r^{2}=1$ in black and no linkage disequilibrium or $r^{2}=0$ in white. Linkage disequilibrium values in between are represented in shades of grey. The panel depicts the linkage disequilibrium structure in a White ethnic group (CEPH from HapMap). rs4713916 was not genotyped by the HapMap consortium. the SNPs had more than $10 \%$ genotyping errors or were in severe Hardy-Weinberg disequilibrium $(P<0.001)$.

\section{Results}

\section{Analysis 1 - general population twin study}

Of the 421 participants with complete data, 19 individuals had to be excluded because of more than $10 \%$ genotyping errors. This resulted in a data-set of 401 participants of White ethnic group with a mean age of 27.7 years (s.d. $=7.9$; range 18-61). A total of $61 \%$ had received higher education, $36.9 \%$ had attended higher secondary school and $2.2 \%$ had completed primary school only. The majority were employed $(62.1 \%)$ and in a relationship $(75.2 \%)$. The mean trauma score was 1.7 (s.d.=0.6). The mean CAPE score was 1.18 (range 1-4.1). There was a significant main effect of trauma in the model of subclinical psychosis $(b=0.41$ (s.e. $=0.08), P<0.001)$, however, no genetic main effect for any of the SNPs ( $P$-values between 0.4 and 0.8). Three SNPs, rs9296158, rs1043805 and rs1360780, showed significant interaction at $P<0.01$ with childhood trauma in the model of subclinical psychosis, and rs4713916 showed suggestive interaction (Table 1 and Table 2).

\section{Analysis 2 - biological validation}

There was no main effect of trauma $(b=-0.03$ (s.e. $=0.04)$, $P=0.41)$ or any of the SNPs ( $P$-values between 0.5 and 0.9$)$ in the model of mean cortisol level. However, there was a significant interaction with trauma for the following FKBP5 SNPs: rs9296158 $\left(\chi^{2}(1)=4.72, P=0.03\right), \operatorname{rs} 4713916\left(\chi^{2}(1)=5.67, P=0.02\right)$ and a trend for $\operatorname{rs} 1360780\left(\chi^{2}(1)=3.35, \quad P=0.067\right)$, but not for rs1043805 $\left(\chi^{2}(1)=0.51, P=0.48\right)$ (Table 2). As depicted in Fig. 2, participants homozygous for the FKBP5 risk alleles who were exposed to higher levels of trauma had significantly lower mean cortisol levels, indicative of increased glucocorticoid receptor sensitivity, whereas this was not found in the participants with lower levels of trauma or with other FKBP5 genotypes.

\section{Analysis 3 - healthy controls}

As described above, the sample for analysis 3 comprised 175 healthy controls. They had a mean age of 30.7 years (s.d.=11.13; range 16-56), $119(68 \%)$ were female, $84 \%$ had received higher education, $14.3 \%$ had attended higher secondary school and $1.7 \%$ had finished primary school only. The ethnicity of the controls was: $93 \%$ White, $6 \%$ mixed and 1\% was from another ethnic group. The mean trauma score was 1.4 (s.d. $=0.4$ ). The mean SIS-R score was 0.50 (range 0-2.14). Allele frequencies were $G / G: 48.8 \%, A / G: 41.2 \%, A / A: 10 \%$ for rs9296158; $G / G: 46.5 \%, A / G: 43 \%, A / A: 10.5 \%$ for rs4713916; $A / A: 68 \%, A / C: 28 \%, C / C: 4 \%$ for rs992105; $G / G: 52.5 \%, G / T$ : $38.5 \%, T / T: 9 \%$ for $\mathrm{rs} 3800373$.

There was no main effect of trauma on subclinical psychosis ( $b=0.01$ (s.e. $=0.02), P=0.66$ ), and none of the SNPs showed a significant main effect ( $P$-values between 0.34 to 0.9 ).

In this sample, none of the four SNPs showed interaction at $P<0.05$ with childhood trauma in the model of subclinical psychosis $\quad($ rs4713916 $\quad(F(1,143)=0.02, \quad P=0.90) ; \quad$ rs9296158 $(F(1,144)=0.08, P=0.77) ; \operatorname{rs} 992105 \quad(F(1,144)=0.12, P=0.73)$; rs3800373 $(F(1,144)=0.24 ; P=0.63))($ Table 2$)$.

\section{Analysis 4 - unaffected siblings}

The final sample of unaffected siblings included 200 individuals with a mean age of 26.58 years (s.d. $=8.06$; range $14-50$ ). There 


\begin{tabular}{|c|c|c|c|c|c|}
\hline SNP and allele frequencies & $\begin{array}{c}\text { Hardy-Weinberg } \\
\text { equilibrium, } P\end{array}$ & Coefficient (b) & $95 \% \mathrm{Cl}$ & $P$ & Risk allele \\
\hline $\begin{array}{r}\text { rs9296158 } \\
\text { G/G: } 48 \% \\
A / G: 40 \% \\
A / A: 12 \% \\
\end{array}$ & 0.08 & 0.33 & 0.09 to 0.57 & 0.007 & A \\
\hline $\begin{array}{l}\text { rs4713916 } \\
\text { G/G: } 47 \% \\
\text { A/G: } 38.5 \% \\
\text { A/A: } 14.5 \% \\
\end{array}$ & 0.006 & 0.20 & -0.03 to 0.43 & 0.08 & A \\
\hline $\begin{array}{c}\text { rs1043805 } \\
\text { A/A: } 68.5 \% \\
\text { A/C: } 29 \% \\
\text { C/C: } 2.5 \% \\
\end{array}$ & 0.68 & 0.66 & 0.31 to 1.01 & 0.0003 & A \\
\hline $\begin{array}{c}\text { rs1360780 } \\
\text { G/G: } 51 \% \\
\text { G/T: } 37.5 \% \\
\text { T/T: } 11.5 \%\end{array}$ & 0.01 & 0.31 & 0.10 to 0.51 & 0.004 & $T$ \\
\hline
\end{tabular}

\begin{tabular}{|c|c|c|c|c|c|c|}
\hline Single nucleotide polymorphisms & $\begin{array}{c}\text { General } \\
\text { population, } \\
\text { psychosis }(n=401)\end{array}$ & $\begin{array}{c}\text { General } \\
\text { population, } \\
\text { cortisol }(n=345)\end{array}$ & $\begin{array}{c}\text { Healthy } \\
\text { controls, psychosis } \\
(n=175)\end{array}$ & $\begin{array}{l}\text { Unaffected } \\
\text { siblings, } \\
\text { psychosis }(n=200)\end{array}$ & $\begin{array}{l}\text { Patients, } \\
\text { psychosis } \\
(n=195)\end{array}$ & Consistency $^{\mathrm{b}}$ \\
\hline rs9296158 & $0.007(A)$ & $0.03(A)$ & 0.77 & 0.32 & $0.03(A)$ & +++ \\
\hline rs4713916 & $0.08(A)$ & $0.02(A)$ & 0.90 & $0.02(A)$ & 0.27 & +++ \\
\hline rs1043805/rs992105 ( $\left.r^{2}=1.0\right)$ & $0.0003(A)$ & 0.48 & 0.73 & $0.04(C)^{\mathrm{c}}$ & 0.20 & +- \\
\hline rs1360780/rs3800373 $\left(r^{2}=0.94\right)$ & $0.004(T)$ & $0.07(T)$ & 0.63 & 0.59 & 0.13 & ++ \\
\hline \multicolumn{7}{|c|}{$\begin{array}{l}\text { a. Table refers to all analysis (1-5). Significant interactions are in bold. } \\
\text { b. Consistency with respect to direction of effects. } \\
\text { c. rs992105 (C) is in perfect linkage with rs1043805 (T). }\end{array}$} \\
\hline
\end{tabular}
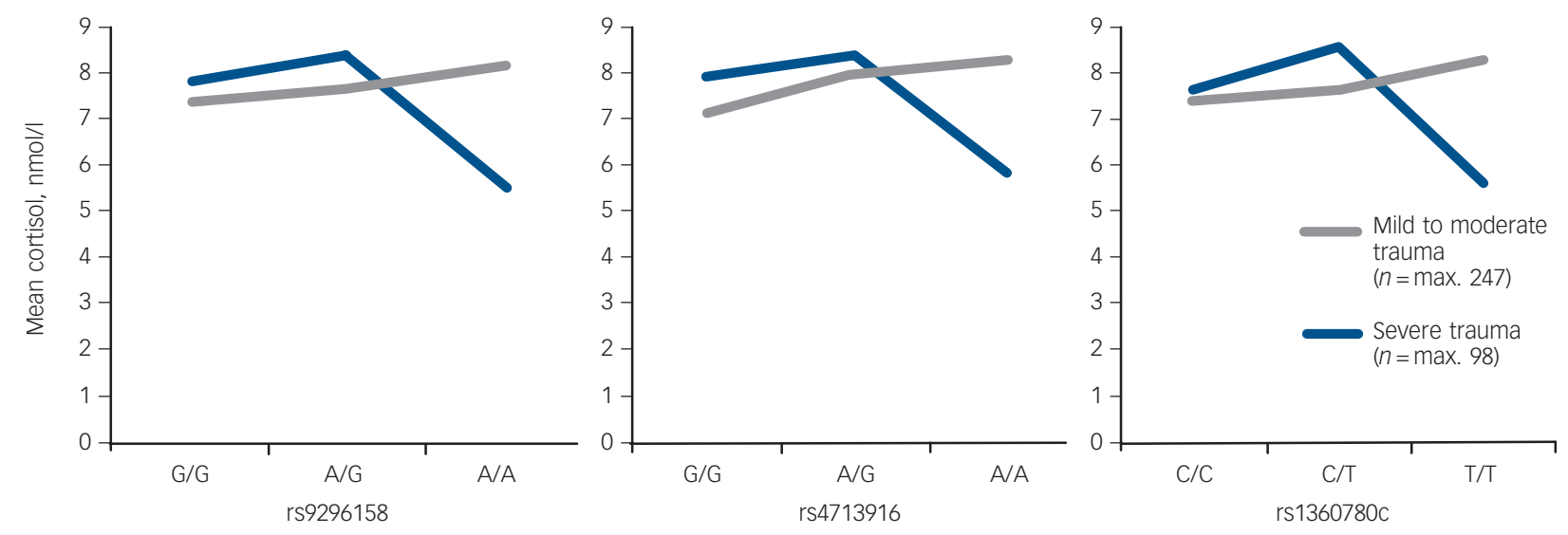

Fig. 2 Mean cortisol level as a function of FK506 binding protein 5 (FKBP5) genotype and level of trauma exposure, indicating glucocorticoid receptor hypersensitivity in traumatised risk genotypes of the general population.

For mild to moderate trauma $n$ was a maximum of 247. For severe trauma $n$ was a maximum of 98 .

were $112(56 \%)$ women, $68 \%$ had received higher education, $22 \%$ had attended higher secondary school and 10\% had finished primary school only The ethnicity of this sample was: $81 \%$ White, $9 \%$ mixed and $2 \%$ from another ethnic group. The mean trauma score was 1.5 (s.d. $=0.4)$. The mean SIS-R score was 0.60 (range $0-2.57$ ). There was no main effect of trauma on subclinical psychosis $(b=0.02$ (s.e. $=0.02), P=0.24)$, and none of the FKBP5 SNPs showed a significant main effect ( $P$-values between 0.4 and 0.8 ).

In this sample, there was a significant interaction with trauma in the model of level of positive schizotypy for rs4713916 $(F(1,166)=5.25, \quad P=0.02) \quad$ and $\operatorname{rs} 992105 \quad(F(1,166)=4.23$, $P=0.04)$, but not for $\operatorname{rs} 9296158(F(1,166)=0.99, P=0.32)$ and rs3800373 $(F(1,163)=0.30, P=0.59)($ Table 2 and Table 3$)$. 


\begin{tabular}{|c|c|c|c|c|c|}
\hline SNP and allele frequencies & $\begin{array}{c}\text { Hardy-Weinberg } \\
\text { equilibrium, } P\end{array}$ & Coefficient $(b)$ & $95 \% \mathrm{Cl}$ & $P$ & Risk allele \\
\hline $\begin{array}{c}\text { rs9296158 } \\
\text { G/G: } 56 \% \\
\text { A/G: } 37 \% \\
\text { A/A: } 7 \%\end{array}$ & 0.60 & 0.03 & -0.03 to 0.10 & 0.32 & - \\
\hline $\begin{array}{c}\text { rs4713916 } \\
\text { G/G: } 56 \% \\
\text { A/G: } 38 \% \\
\text { A/A: } 6 \%\end{array}$ & 0.20 & 0.07 & 0.10 to 0.14 & 0.02 & A \\
\hline $\begin{array}{c}\text { rs992105 } \\
\text { A/A: } 75 \% \\
\text { A/C: } 23 \% \\
\text { C/C: } 2 \%\end{array}$ & 0.38 & 0.08 & 0.003 to 0.16 & 0.04 & c \\
\hline $\begin{array}{c}\text { rs3800373 } \\
\text { G/G: } 59 \% \\
\text { G/T: } 35 \% \\
\text { T/T: } 6 \%\end{array}$ & 0.30 & 0.02 & -0.05 to 0.09 & 0.59 & - \\
\hline
\end{tabular}

\begin{tabular}{|c|c|c|c|c|c|}
\hline SNP and allele frequencies & $\begin{array}{l}\text { Hardy-Weinberg } \\
\text { equilibrium, } P\end{array}$ & Coefficient (b) & $95 \% \mathrm{Cl}$ & $P$ & Risk allele \\
\hline $\begin{array}{c}\text { rs9296158 } \\
\text { G/G: } 47 \% \\
\text { A/G: } 44 \% \\
\text { A/A: } 9 \%\end{array}$ & 0.60 & 0.07 & 0.005 to 0.13 & 0.03 & A \\
\hline $\begin{array}{r}r 4713916 \\
\text { G/G: } 52 \% \\
\text { A/G: } 36 \% \\
A / A: 12 \%\end{array}$ & 0.20 & 0.03 & -0.024 to 0.086 & 0.27 & - \\
\hline $\begin{array}{c}r S 992105^{\mathrm{b}} \\
\text { A/A: } 69 \% \\
\text { A/C: } 29 \% \\
\text { C/C: } 2 \% \\
\end{array}$ & 0.38 & 0.06 & -0.03 to 0.14 & 0.20 & \\
\hline $\begin{array}{c}\text { rs3800373 } \\
\text { G/G: } 51 \% \\
\text { G/T: } 42 \% \\
\text { T/T: } 7 \%\end{array}$ & 0.30 & 0.05 & -0.01 to 0.12 & 0.13 & - \\
\hline
\end{tabular}

\section{Analysis 5 - association with symptoms in patients}

The final patient sample included 195 patients with a psychotic disorder with a mean age of 28.1 years (s.d. $=8.3$; range $15-60$ ). There were 52 women $(26.7 \%)$ and $58.5 \%$ had received higher education, $30.0 \%$ had attended higher secondary school and $11.5 \%$ had finished primary school only. The ethnicity of the patient sample was: $87 \%$ White, $9 \%$ mixed ethnicity and $4 \%$ from another ethnic group. The mean trauma score was 1.7 (s.d.=0.6). The mean BPRS score was 1.67 (range 1-4.60). There was a significant main effect of trauma on BPRS score $(b=0.06$ $($ s.e. $=0.02), P<0.005)$, and there were no genetic main effects $(P$-values between 0.2 and 0.9$)$.

One SNP moderated the effect of trauma on psychotic symptoms (measured with the BPRS) in patients with a psychotic disorder: $\operatorname{rs} 9296158(F(1,161)=4.55, \quad P=0.03)$, whereas no interaction was found for $\operatorname{rs} 4713916(F(1,158)=1.24, P=0.27)$, rs992105 $(F(1,160)=1.69, P=0.20)$ and $\operatorname{rs3800373}(F(1,158)=$ 2.38, $P=0.13$ ) (Table 2 and Table 4 ).

Results that were partially consistent over all analyses were thus found for rs9296158 and rs4713916: significant, directionally similar interactions were found for both of these SNPs in at least two independent samples in combination with an interaction in the same direction for a biological/physiological outcome measure; however, inconclusive results were also found (Table 2).

\section{Discussion}

Given the pivotal role of FKBP5 in moderating stress response and risk for PTSD and depression, the current paper, for the first time, examined the role of FKBP $5 \times$ trauma interactions in psychosis. The most consistent finding was an interaction between two FKBP5 SNPs, namely rs9296158 and rs4713916, and childhood 
trauma in the models of both psychotic symptoms and cortisol, showing robust effects in the general population twin sample, in combination with a directionally similar interaction in unaffected siblings (rs4713916) and patients with a psychotic disorder (rs9296158).

\section{FKBP5, cortisol, dopamine and psychosis}

Our results are in line with other human and animal studies, supporting the role of the FKBP5 genotype in modifying the effects of childhood trauma on mental disorders, ${ }^{11,20,28-31}$ although the implicated SNPs were not always the same. Specifically, the two SNPs most consistently interacting with childhood trauma in the present study (rs9296158/rs4713916) were also found to interact in previous studies of PTSD, ${ }^{28}$ suicidality $^{32}$ and depression, ${ }^{31}$ whereas in another study these SNPs did not significantly interact with trauma ${ }^{29}$ or were not investigated. ${ }^{20}$ The two remaining SNPs that did not show convincing evidence for interaction in this study (rs992105/ rs1043805 and rs3800373/rs1360780) were previously found to interact with trauma in other studies examining adult PTSD symptoms, ${ }^{28}$ peri-traumatic dissociation in children after medical trauma ${ }^{33}$ and depression. ${ }^{31}$ Reasons for inconsistent interactions, at the level of the specific SNPs implied, may be the large degree of linkage disequilibrium in FKBP5 (as depicted in Fig. 1) and the limited knowledge about the functional properties of these SNPs. ${ }^{11}$

Previous work has shown that individuals with FKBP5 highinduction alleles have a greater risk of developing PTSD following childhood trauma. ${ }^{28}$ Furthermore, whereas high-induction alleles were associated with relative glucocorticoid receptor resistance in individuals without PTSD, there was a reversal of this association in individuals exposed to childhood trauma; high-induction genotypes with a diagnosis of PTSD displaying glucocorticoid receptor supersensitivity rather than glucocorticoid receptor resistance. ${ }^{28}$ Our results were similar in this regard: exposure to childhood trauma seemingly induced glucocorticoid receptor supersensitivity in individuals with high-induction alleles as evidenced by lower mean cortisol levels. Additionally, traumatised individuals with high-induction alleles showed higher levels of psychosis expression. Phenomenological similarities between PTSD and psychotic disorder may thus share a common underlying mechanism. Therefore, carefully enquiring about trauma history in patients with psychosis is useful, allowing targeted interventions to reduce trauma-related anxiety, hyperarousal and stress-sensitivity in these patients. ${ }^{34}$

There is biological plausibility to the hypothesis that individuals homozygous for the high-induction alleles carry the highest risk for psychosis expression and decreased cortisol levels (as a result of glucocorticoid receptor hypersensitivity) when exposed to trauma. After cortisol binding, the glucocorticoid receptor translocates from the cytosol to the nucleus where it exerts its effects on glucocorticoid receptor-sensitive genes. Glucocorticoid receptor sensitivity can be modulated by molecules binding to this system, so-called 'co-chaperones', one of which is FKBP5. Upon cortisol binding, FKBP5 is replaced by FKBP4, which allows for the translocation of the glucocorticoid receptor complex into the nucleus. There, the glucocorticoid receptor complex then increases FKBP5 translation, which in turn confers higher glucocorticoid receptor resistance in the cytosol, thus ensuring a direct feedback loop on glucocorticoid receptor sensitivity. ${ }^{11}$

Our results suggest that trauma exposure, most likely due to an excess of cortisol exposure in childhood, may have an impact on these mechanisms, thereby resulting in increased glucocorticoid receptor hypersensitivity in individuals homozygous for the high-induction alleles. In support of this hypothesis is a study showing that mice lacking the gene encoding FKBP5 were shown to be less vulnerable to the adverse effects of social defeat. ${ }^{35}$ Rodent work also suggests that trauma results in increased methylation of the glucocorticoid receptor ${ }^{36}$ and maternal behaviour changed HPA axis responses to stress by regulating glucocorticoid receptor gene expression through epigenetic programming in rodents ${ }^{37}$ and humans. ${ }^{38}$ These mechanisms may be regulated by FKBP5 polymorphisms, as suggested by work in mice showing that a combined FKBP5 deletion and additional stress exposure resulted in decreased HPA axis reactivity and glucocorticoid receptor expression. ${ }^{39}$

Furthermore, dysregulation of the HPA axis - including dysregulation of cortisol and possibly also abnormalities in glucocorticoid receptors - was repeatedly demonstrated in patients with a psychotic disorder. ${ }^{7}$ Although the exact mechanisms remain to be elucidated, evidence suggests that glucocorticoid secretion may increase dopamine activity in brain regions that have been associated with the experience of positive psychotic symptoms. ${ }^{3}$ Importantly, glucocorticoid receptor sensitivity influences in vivo dopamine release in response to stress in the rat prefrontal cortex, ${ }^{40}$ providing credence to the notion that FKBP5 may contribute to the onset of psychotic experiences in the context of exposure to childhood trauma.

\section{Strengths and shortcomings}

Although the present results may have considerable biological plausibility, it is important to cautiously consider the limitations. The most important consideration in this regard is the risk of false-positive findings associated with any genetic study, especially in the context of undisclosed statistical comparisons, low a priori probability of interaction and/or low statistical power, ${ }^{41}$ issues that are more common in (but are not exclusive to) candidate gene approaches. We have carefully considered these issues in the present study. First, we would argue that the a priori probability of interaction is relatively high, given (a) consistent findings of an FKBP $5 \times$ trauma interaction in PTSD and other stress-related phenotypes, and (b) considerable biological plausibility, which was supported by the finding of an FKBP $5 \times$ trauma interaction for cortisol, thus linking FKBP5dependent psychometric expression of psychotic symptoms to an underlying biological mechanism. Taken together, this allowed us to pursue a hypothesis-driven $\mathrm{G} \times \mathrm{E}$ interaction approach using functional polymorphisms, thus limiting the number of statistical tests. It has been argued that this may be the most meaningful approach towards $\mathrm{G} \times \mathrm{E}$ interactions, ${ }^{42}$ although other approaches, in particular those incorporating information from genome-wide studies, may also be valuable for future research. ${ }^{13}$ Second, power analyses based on previous $\mathrm{FKBP} 5 \times$ trauma literature in other stress-related phenotypes, ${ }^{20}$ as well as on a recent study reporting interaction between a functional polymorphism in brain-derived neurotrophic factor (BDNF) and childhood trauma in models of adult psychotic experiences in the general population, ${ }^{21}$ indicated fair (analysis 4) to excellent (analysis 1,2,5) power, whereas power was modest for analysis 3. However, further validation and replication of the present findings is clearly still needed, especially since the findings in the follow-up samples were not unambiguous. One reason for this could be that our power estimations were based on studies without a replication sample. As it is known that effect sizes are usually larger in discovery samples than in subsequent replication efforts, we may have overestimated the 'true' effect size. The observation that the greatest number of significant interactions was found in the best-powered analyses may be in agreement with this view, and in support of our interpretation that the present findings are 
unlikely to be spurious. It should be noted that we were only able to test the effect of the trauma $\times$ FKBP5 interaction on development of psychotic symptoms along the psychosis continuum, but were unable to examine a differential effect on psychotic diagnostic outcome because of the low power for the case-only design. Future studies should incorporate clinical outcomes.

Some other limitations should be mentioned. The detection samples, for instance, consisted of women only. This may be important in the light of the high prevalence of affective symptoms in young adult females and the co-occurrence of affective and psychotic symptoms. However, as all analyses were controlled for depressive symptoms, it is unlikely that this would explain the results. In the twin sample, two (out of four) SNPs were also in moderate Hardy-Weinberg disequilibrium, one of which produced a significant result. As SNPs in Hardy-Weinberg disequilibrium are less powerful, and do not tend to increase false-positive results, ${ }^{43}$ the reported results are unlikely to be caused by Hardy-Weinberg disequilibrium, especially given the absence of quality control issues (in our samples the genotypes were successfully identified at rates (call rates) of between 95 and $98 \%$ ). Also, this study focused solely on the circulating hormone cortisol and did not include dexamethasone-corticotropin-releasing hormone or dexamethasone suppression tests ${ }^{11}$ and can thus provide no ultimate insight into underlying mechanisms at higher levels of the HPA axis. However, we included repeated measures of salivary cortisol over 5 days (on average about 38 cortisol measures per individual), thereby taking into account the wellknown but often ignored unreliability of cortisol measures obtained at infrequent intervals. ${ }^{44}$

\footnotetext{
Dina Collip, PhD, Inez Myin-Germeys, PhD, Marieke Wichers, PhD, Department of Psychiatry and Psychology, School of Mental Health and Neuroscience, Maastricht University Medical Centre, Maastricht, The Netherlands, Nele Jacobs, PhD, Department of Psychiatry and Psychology, School of Mental Health and Neuroscience, Maastricht University Medical Centre, Maastricht, The Netherlands; Catherine Derom, PhD, Department of Human Genetics, University Hospital Gasthuisberg, Catholic University Leuven, Belgium; Evert Thiery, PhD, Department of Neurology, Ghent University Hospital, Ghent, Belgium; Tineke Lataster, PhD, Claudia Simons, Ghent University Hospital, Ghent, Belgium; Tineke Lataster, PhD, Claudia Simons,
PhD, Philippe Delespaul, PhD, Machteld Marcelis, MD, PhD, Department of Psychiatry and Psychology, School of Mental Health and Neuroscience, Maastricht University Medical Centre, Maastricht, The Netherlands; Jim van Os, PhD, Department of Psychiatry and Psychology, School of Mental Health and Neuroscience, Maastricht University Medical Centre, Maastricht, The Netherlands and King's College van Winkel, MD, PhD, Department of Psychiatry and Psychology, School of Mental van Winkel, MD, PhD, Department of Psychiatry and Psychology, School of Mentricht,
Health and Neuroscience, Maastricht University Medical Centre, Maastricht, The Netherlands and University Psychiatric Centre Catholic University Leuven, Kortenberg Belgium
}

Correspondence: Ruud van Winkel, Department of Psychiatry and Psychology, School of Mental Health and Neuroscience, Maastricht University Medical Centre, PO Box 616 (DRT 10), 6200 MD Maastricht, The Netherlands. Email: ruud.vanwinkel@sp.unimaas.nl

First received 5 Jun 2012, final revision 15 Oct 2012, accepted 17 Dec 2012

\section{Funding}

The TWIN study has been partly supported by grants from the Fund of Scientific Research, Flanders and Twins, a non-profit association for Scientific Research in Multiple Birth (Belgium). The infrastructure for the GROUP study is funded by the Geestkracht programme of the Dutch Health Research Council (ZON-MW, grant number 10-000-1002) and matching funds from participating universities and mental health care organisations (Amsterdam: Academic Psychiatric Centre AMC, Ingeest, Arkin, Dijk en Duin, Rivierduinen, Erasmus MC, GGZ Noord Holland Noord; Utrecht: University Medical Centre Utrecht, Altrecht, MC, GGZ Noord Holland Noord; Utrecht: University Medical Centre Utrecht, Altrecht,
Symfora, Meerkanten, Riagg Amersfoort, Delta; Groningen: University Medical Center Groningen, Lentis, GGZ Friesland, GGZ Drenthe, Dimence, Mediant, GGZ De Grote Rivieren and Parnassia Bavo Groep; Maastricht: Maastricht University Medical Center, GGZ Eindhoven, GGZ Midden-Brabant, GGZ Oost-Brabant, GGZ Noord- Midden Limburg, Mondriaan Zorggroep, Prins Clauscentrum Sittard, RIAGG Roermond, Universitair Centrum Sint-Jozef Kortenberg CAPRI University of Antwerp, PC Ziekeren Sint-Truiden, PZ Sancta Maria Sint-Truiden, GGZ Overpelt, OPZ Rekem). The research leading to these results has Maria Sint-Truiden, GGZ Overpelt, OPZ Rekem). The research leading to these results has
received funding from the European Community's Seventh Framework Program under grant agreement No. HEALTH-F2-2009-241909 (Project EU-GEI).

\section{Acknowledgements}

We are grateful for the generosity of time and effort by the families who made the GROUP project possible. The GROUP investigators comprise: René S. Kahn (Rudolf Magnus Institute of Neuroscience, Utrecht, The Netherlands), Don H. Linszen (University of Amsterdam, The Netherlands), Jim van Os (Maastricht University Medical Centre, The Netherlands and Institute of Psychiatry, London, UK), Durk Wiersma, Richard Bruggeman (University of Groningen, The Netherlands), Wiepke Cahn (Rudolf Magnus Institute of Neuroscience, Utrecht, The Netherlands), Lieuwe de Haan (University of Amsterdam, The Netherlands), Lydia Krabbendam, Inez Myin-Germeys (Maastricht University Medical Centre, The Netherlands).

\section{References}

1 Varese F, Smeets F, Drukker M, Lieverse R, Lataster T, Viechtbauer W, et al. Childhood adversities increase the risk of psychosis: a meta-analysis of patient-control, prospective-and cross-sectional cohort studies. Schizophr Bull 2012; 38: 661-71.

2 Heins $M$, Simons $C$, Lataster $T$, Pfeifer $S$, Versmissen $D$, Lardinois $M$, et al. Childhood trauma and psychosis: a case-control and case-sibling comparison across different levels of genetic liability, psychopathology, and type of trauma. Am J Psychiatry 2011; 168: 1286-94.

3 van Winkel R, Stefanis NC, Myin-Germeys I. Psychosocial stress and psychosis. A review of the neurobiological mechanisms and the evidence for gene-stress interaction. Schizophr Bull 2008; 34: 1095-105.

4 Read J, Perry BD, Moskowitz A, Connolly J. The contribution of early traumatic events to schizophrenia in some patients: a traumagenic neurodevelopmental model. Psychiatr Interpers Biol Process 2001; 64: 319-45.

5 Collip D, Nicolson N, Lardinois M, Lataster T, van Os J, Myin-Germeys I. Daily cortisol, stress reactivity and psychotic experiences in individuals at above average genetic risk for psychosis. Psychol Med 2011; 41: 2305-15.

6 Aiello G, Horowitz M, Hepgul N, Pariante CM, Mondelli V. Stress abnormalities in individuals at risk for psychosis: a review of studies in subjects with familial risk or with "at risk" mental state. Psychoneuroendocrinology 2012; 37: 1600-13

7 Walker EF, Walder DJ, Reynolds F. Developmental changes in cortisol secretion in normal and at-risk youth. Dev Psychopathol 2001; 13: 721-32.

8 Zammit S, Owen MJ, Evans J, Heron J, Lewis G. Cannabis, COMT and psychotic experiences. Br J Psychiatry 2011; 199: 380-5.

9 Karg K, Burmeister M, Shedden K, Sen S. The serotonin transporter promoter variant (5-HTTLPR), stress, and depression meta-analysis revisited: evidence of genetic moderation. Arch Gen Psychiatry 2011; 68: 444-53.

10 Koenen K, Saxe G, Purcell S, Smoller J, Bartholomew D, Miller A, et al. Polymorphisms in FKBP5 are associated with peritraumatic dissociation in medically injured children. Mol Psychiatry 2005; 10: 1058-9.

11 Binder EB. The role of FKBP5, a co-chaperone of the glucocorticoid receptor in the pathogenesis and therapy of affective and anxiety disorders. Psychoneuroendocrinology 2009; 34 (suppl 1): S186-95.

12 Kelleher I, Cannon M. Psychotic-like experiences in the general population: characterizing a high-risk group for psychosis. Psych Med 2011; 41: 1.

13 Van Winkel R, Esquivel G, Kenis G, Wichers M, Collip D, Peerbooms O, et al. REVIEW: Genome-wide findings in schizophrenia and the role of geneenvironment interplay. CNS Neurosci Ther 2010; 16: e185-92.

14 Derom C, Vlietinck R, Thiery E, Leroy F, Fryns JP, Derom R. The East Flanders Prospective Twin Survey (EFPTS). Twin Res 2002; 5: 337-41.

15 First MB, Spitzer RL, Gibbon M, Williams JB. Structured Clinical Interview for DSM-IV Axis I Disorders. New York State Psychiatric Institute, 1995.

16 Konings M, Bak M, Hanssen M, van Os J, Krabbendam L. Validity and reliability of the CAPE: a self-report instrument for the measurement of psychotic experiences in the general population. Acta Psychiatr Scand 2006; 114: 55-61.

17 Bernstein DP, Stein JA, Newcomb MD, Walker E, Pogge D, Ahluvalia T, et al. Development and validation of a brief screening version of the Childhood Trauma Questionnaire. Child Abuse Neg/ 2003; 27: 169-90.

18 Jacobs N, Myin-Germeys I, Derom C, Delespaul P, van Os J, Nicolson NA A momentary assessment study of the relationship between affective and adrenocortical stress responses in daily life. Biol Psychol 2007; 74: 60-6.

19 Dressendörfer R, Kirschbaum C, Rohde W, Stahl F, Strasburger C. Synthesis of a cortisol-biotin conjugate and evaluation as a tracer in an immunoassay for salivary cortisol measurement. J Steroid Biochem Mol Biol 1992; 43: 683-92.

20 Appel K, Schwahn C, Mahler J, Schulz A, Spitzer C, Fenske K, et al. Moderation of adult depression by a polymorphism in the FKBP5 gene and childhood physical abuse in the general population. Neuropsychopharmacology 2011; 36: 1982-91. 
21 Alemany S, Arias B, Aguilera M, Villa H, Moya J, Ibáñez MI, et al. Childhood abuse, the BDNF-Val66Met polymorphism and adult psychotic-like experiences. Br J Psychiatry 2011; 199: 38-42.

22 National Institute of Mental Health. Genetics. Initiative. Family Interview for Genetic Studies (FIGS). NIMH, 1992.

23 American Psychiatric Association. Diagnostic and Statistical Manual of Mental Disorder (4th edn) (DSM-IV). APA, 1994.

24 Andreasen NC, Flaum M, Arndt S. The Comprehensive Assessment of Symptoms and History (CASH). An instrument for assessing diagnosis and psychopathology. Arch Gen Psychiatry 1992; 49: 615-23.

25 Kendler KS, Lieberman JA, Walsh D. The Structured Interview 559 for Schizotypy (SIS): a preliminary report. Schizophr Bull 1989; 15: 559-71.

26 Thomas A, Donnell AJ, Young TR. Factor structure and differential validity of the expanded Brief Psychiatric Rating Scale. Assessment 2004; 11: 177-87.

27 van Winkel R. Family-based analysis of genetic variation underlying psychosis-inducing effects of cannabis: sibling analysis and proband follow-up. Arch Gen Psychiatry 2011; 68: 148-57.

28 Binder EB, Bradley RG, Liu W, Epstein MP, Deveau TC, Mercer KB, et al. Association of FKBP5 polymorphisms and childhood abuse with risk of posttraumatic stress disorder symptoms in adults. JAMA 2008; 299: 1291-305.

29 Xie $\mathrm{P}$, Kranzler HR, Poling J, Stein MB, Anton RF, Farrer LA, et al. Interaction of FKBP5 with childhood adversity on risk for post-traumatic stress disorder. Neuropsychopharmacology 2010; 35: 1684-92.

30 Velders FP, Kuningas M, Kumari M, Dekker MJ, Uitterlinden AG, Kirschbaum $\mathrm{C}$, et al. Genetics of cortisol secretion and depressive symptoms: a candidate gene and genome wide association approach. Psychoneuroendocrinology 2011; 36: 1053-61.

31 Zimmermann $\mathrm{P}$, Brückl T, Nocon A, Pfister $\mathrm{H}$, Binder EB, Uhr $\mathrm{M}$, et al. Interaction of FKBP5 gene variants and adverse life events in predicting depression onset: results from a 10-year prospective community study. Am J Psychiatry 2011; 168: 1107-16.

32 Roy A, Gorodetsky E, Yuan Q, Goldman D, Enoch MA. Interaction of FKBP5, a stress-related gene, with childhood trauma increases the risk for attempting suicide. Neuropsychopharmacology 2010; 35: 1674-83.

33 Koenen KC, Uddin M. FKBP5 polymorphisms modify the effects of childhood trauma. Neuropsychopharmacology 2010; 35: 1623-24.
34 Read J, Hammersley P, Rudegeair T. Why, when and how to ask about childhood abuse. Adv Psychiatr Treat 2007; 13: 101-10.

35 Hartmann J, Wagner KV, Dedic N, Marinescu D, Scharf SH, Wang XD, et al. Fkbp52 heterozygosity alters behavioral, endocrine and neurogenetic parameters under basal and chronic stress conditions in mice. Psychoneuroendocrinology 2012; 37: 2009-12.

36 McGowan PO, Sasaki A, D’Alessio AC, Dymov S, Labonté B, Szyf M, et al. Epigenetic regulation of the glucocorticoid receptor in human brain associates with childhood abuse. Nat Neurosci 2009; 12: 342-48.

37 Weaver ICG, Cervoni N, Champagne FA, D'Alessio AC, Sharma S, Seckl JR, et al. Epigenetic programming by maternal behavior. Nat Neurosci 2004; 7 $847-54$.

38 Champagne FA, Curley JP. Epigenetic mechanisms mediating the long-term effects of maternal care on development. Neurosci Biobehav Rev 2009; 33: 593-600.

39 Touma C, Gassen NC, Herrmann L, Cheung-Flynn J, Büll DR, Ionescu IA et al. FK506 binding 5 shapes stress responsiveness: modulation of neuroendocrine reactivity and coping behavior. Biol Psychiatry 2011 15: $928-36$.

40 Butts KA, Weinberg J, Young AH, Phillips AG. Glucocorticoid receptors in the prefrontal cortex regulate stress-evoked dopamine efflux and aspects of executive function. PNAS 2011; 108: 18459-64.

41 Duncan LE, Keller MC. A critical review of the first 10 years of candidate gene-by-environment interaction research in psychiatry. Am J Psychiatry 2011: 168: 1041-9.

42 Moffitt $T E$, Caspi A, Rutter M. Strategy for investigating interactions between measured genes and measured environments. Arch Gen Psychiatry 2005; 62: 473-81.

43 Fardo DW, Becker KD, Bertram L, Tanzi RE, Lange C. Recovering unused information in genome-wide association studies: the benefit of analyzing SNPS out of Hardy-Weinberg equilibrium. Eur J Hum Genet 2009; 17 1676-82.

44 Hruschka DJ, Kohrt BA, Worthman CM. Estimating between- and within-individual variation in cortisol levels using multilevel models. Psychoneuroendocrinology 2005; 30: 698-714.

\section{0 words}

\section{Art Brut}

Rachael Hall

Art Brut, translated from the French as 'raw art', is used to describe art occurring outside of the conventional art scene, typically without the process of formal training that renders art 'cooked'. The term was coined by the French artist Jean Dubuffet in the 1940s. A significant contribution to this genre, although not exclusively, is from those people with exceptional artistic abilities who also suffer from serious mental illness. Free from the constraints of formal art culture, Art Brut allows for unbridled creative expression, in turn giving the viewer a direct insight into the artist's perceptions, fantasies, hopes and fears. 